\title{
Schwannoma del nervio facial y sus ramas en el oído medio: Reporte de 3 casos y revisión de literatura
}

\section{Schwannoma of the facial nerve and its branches in the middle ear: A report of 3 cases and literature review}

\author{
Lara Fernández $\mathbf{R}^{1}$, Macarena Karle $\mathbf{P}^{2}$, Carlos Stott $\mathbf{C}^{1}$.
}

\begin{abstract}
RESUMEN
El schwannoma del nervio facial (SNF) es un tumor infrecuente, sin embargo es el tumor más frecuente del nervio facial. Son tumores benignos de crecimiento lento que producen sintomatología otológica no específica y pueden asociar parálisis facial. No existen exámenes preoperatorios que nos permitan diagnosticar con certeza el SNF. El estudio de elección se realiza con TC de alta resolución y RM con gadolinio. El diagnóstico definitivo es histopatológico pero se puede hacer diagnóstico presuntivo si la clínica, imágenes y hallazgo intraoperatorios son concordantes. El objetivo del tratamiento debe intentar preservar la función del nervio facial por el mayor tiempo posible. En casos de pacientes asintomáticos, o sin compromiso del nervio facial, se prefiere la observación. Se presentan en este artículo casos de schwannomas del facial intratimpánico y de cuerda del tímpano.
\end{abstract}

Palabras claves: Schwannoma, nervio facial, cuerda del tímpano.

\begin{abstract}
The facial nerve schwannoma (SNF) is a rare tumor, however it is the most common tumor of the facial nerve. They are slow-growing benign tumors that produce non-specific otologic symptoms and may be associated facial paralysis. There are no preoperative tests that allow us to accurately diagnose the SNF. The study of choice is made with high resolution CT and MRI with gadolinium. Definitive diagnosis is histopathological but the physician may make a presumptive diagnosis if clinical images and intraoperative findings are consistent. The goal of treatment should try to preserve facial nerve function for as long as possible. In cases of asymptomatic patients, without facial nerve involvement, observation is prefered.

Cases of schwannomas of intratympanic facial and chorda tympani are presented in this article.
\end{abstract}

Key words: Schwannoma, facial nerve, chorda tympani.

\footnotetext{
${ }^{1}$ Médico Otorrinolaringólogo ORL Hospital Clínico Universidad de Chile.

${ }^{2}$ Médico Cirujano, CESFAM Gabriela Mistral.
}

Recibido el 31 de julio, 2014. Aceptado el 5 de septiembre, 2014. 


\section{INTRODUCCIÓN}

Los schwannomas, neurinomas o neurilemomas son tumores benignos originados de las células de Schwann. Representan cerca del $8 \%$ de los tumores intracraneales primarios ${ }^{1}$. El schwannoma del nervio facial (SNF) es un tumor infrecuente, sin embargo es el tumor más frecuente del nervio facial. Los schwannomas se Iocalizan habitualmente en nervios sensitivos, siendo el más frecuente el neurinoma del acústico². Son tumores de crecimiento lento y predecible, generalmente asintomáticos ${ }^{3}$. Aparecen en edades medias de la vida, con una ligera predilección por el sexo femenino. Pese a su carácter benigno, estos tumores causan daño por compresión y/o remodelación de estructuras adyacentes. Los schwannomas originados en las ramas del nervio facial son aún más infrecuentes. A continuación se presentan 2 casos de schwannomas de nervio facial intratemporal y un caso con compromiso de la cuerda del tímpano.

\section{CASOS CLÍNICOS}

\section{Caso 1}

Paciente de 45 años, con 9 años de evolución de parálisis facial, hipoacusia derecha y vértigo ocasional. Al examen se aprecia una masa blanquecina retrotimpánica que abombaba la membrana timpánica (Figura 1). Se solicita tomografía computarizada (TC) y resonancia magnética (RM) de oídos compatibles con tumor de las porciones timpánica y estilomastoídea del nervio facial derecho que ocupa toda la caja y se extiende a mastoides. La audiometría objetiva hipoacusia de conducción derecha. Vía retroauricular se reseca en su totalidad el tumor que se extiende desde el primer codo hasta la porción mastoídea del facial (Figura 2). El estudio anatomopatológico e inmunohistoquímico de la pieza quirúrgica fue positiva para S-100 en células fusadas, concordante con neurinoma.

\section{Caso 2}

Paciente de 39 años, consulta por hipoacusia derecha de 3 años de evolución como único síntoma. Al examen bajo otomicroscopía destaca un tímpano no perforado que deja ver por transparencia una masa blanquecino-parduzca en cuadrante posterosuperior. La audiometría evidencia hipoacusia mixta del oído derecho con un promedio tonal puro (PTP) aéreo de 41,6 dB y un PTP óseo de $23 \mathrm{~dB}$, la impedanciometría muestra una curva $\mathrm{B}$ para el oído afectado con reflejo negativo. La TC de oídos objetiva una masa homogénea en región posterosuperior de la

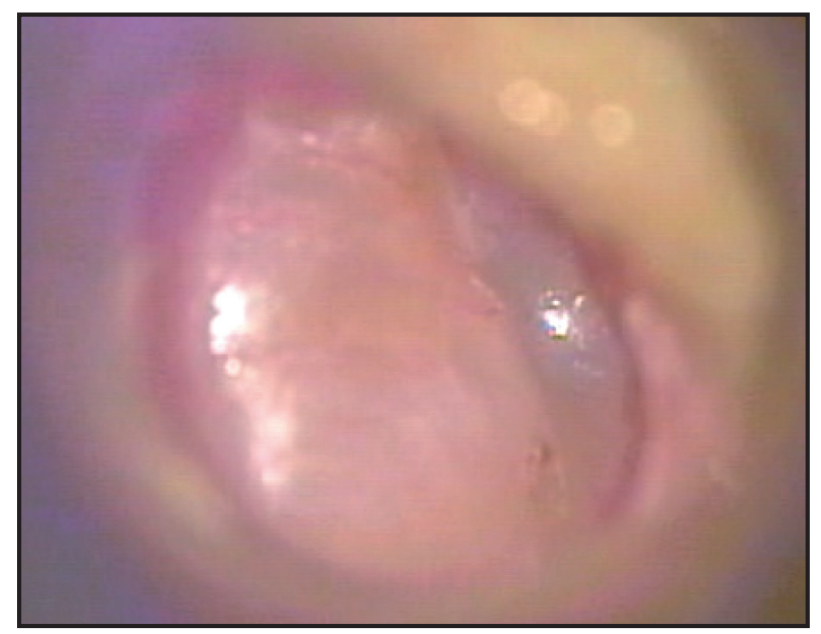

Figura 1. Imagen de la otoscopía, se ve una masa blanquecina retrotimpánica que abomba la membrana timpánica. 


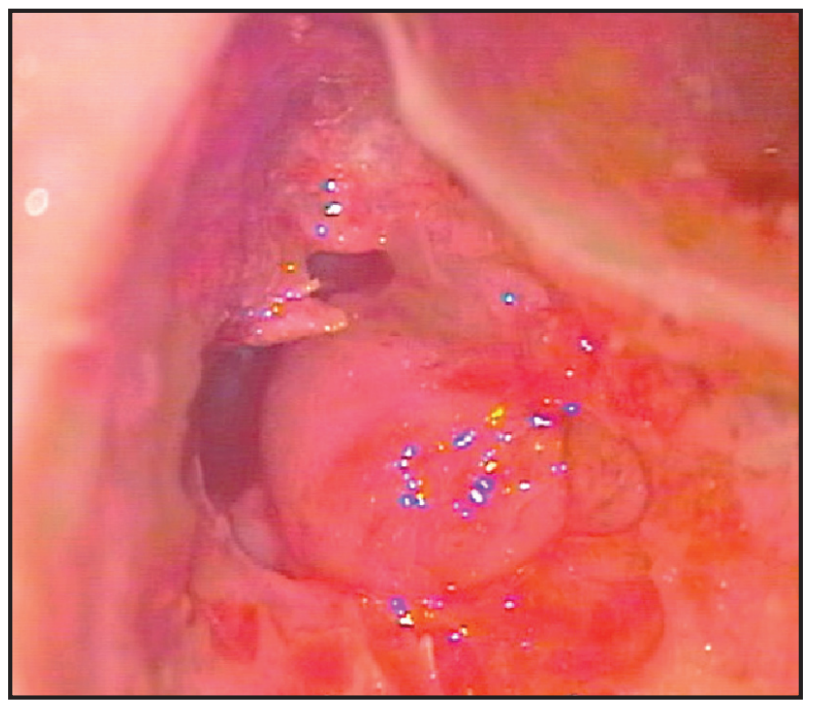

Figura 2. Imagen quirúrgica del tumor vía retroauricular.

cavidad timpánica, que englobaba los huesecillos, sin evidencias de destrucción ósea y en directa relación con la porción horizontal del nervio facial. Se solicita RM la cual ratifica los hallazgos tomográficos, y evidencia que la lesión se contrasta con gadolinio y era hipointensa en la secuencia T2. Se realiza una timpanotomía exploradora en que encuentra que el tumor hacía cuerpo con la cuerda del tímpano, sin relación con el nervio facial ni el promontorio. Se logra extraer la masa en su totalidad, para lo cual fue necesario cortar la cuerda del tímpano y desarticular la cadena, resecando el yunque. El estudio anatomopatológico reveló tejido compuesto por fascículos nerviosos inmersos en tejido cicatrizal fibroso, sin elementos de carácter maligno. La inmunohistoquímica fue positiva para S-100, diagnosticándose un neurinoma de la cuerda del tímpano.

\section{Caso 3}

Paciente de 14 años sin antecedentes mórbidos previos, consulta por historia de 8-10 años de evolución de hipoacusia progresiva en oído izquierdo (OI), sin otros síntomas otológicos. Al examen físico destaca una masa rojiza en cuadrante posterosuperior. Se inicia el estudio con una audiometría que evidencia hipoacusia de conducción en OI con PTP aéreo de 58,3 dB y un PTP óseo de $11,6 \mathrm{~dB}$, la impedanciometría muestra una curva $B$ en oído afectado con reflejo acústico negativo. Se solicita TC en el que informa una malformación de la cadena de huesecillos y nervio facial prominente. Posteriormente se realiza una timpanotomía exploradora donde se encuentra una masa amarillorojiza de aspecto neurofibromatoso en relación al segundo codo del nervio facial. Se decide no resecar el tumor debido a su íntima relación con el nervio, posible origen en tejido nervioso y potencial riesgo de lesión de éste. En el intraoperatorio se confirma que la cadena de huesecillos se encontraba indemne. Actualmente el paciente persiste con hipoacusia pero con función del facial normal.

\section{DISCUSIÓN}

El SNF se puede clasificar de acuerdo al sitio de origen: intracraneal (cisternal), intratemporal (intracanalicular, laberíntico, timpánico y mastoideo) y extracraneal (intraparotídeo). Puede desarrollarse en cualquier segmento del nervio, desde el ángulo pontocerebeloso, hasta las ramas periféricas de la cara, siendo característico que 
involucre múltiples segmentos a la vez, dentro de los cuales los más afectados son el área perigeniculada, segmento laberíntico y timpánico ${ }^{4}$. La presentación clínica varía de acuerdo al sitio comprometido, siendo los síntomas más comunes la hipoacusia de conducción, tinnitus, parálisis facial, vértigo, otorrea y dolor en el caso de los SNF intratemporales y como masa parotídea en los extratemporales ${ }^{1}$.

Dentro de los diagnósticos diferenciales de SNF intratemporal se deben considerar: colesteatoma, meningioma, meningocele, adenoma, tumor glómico, hemangioma y tumores malignos ${ }^{1}$.

Actualmente, no existen exámenes preoperatorios que nos permitan diagnosticar con certeza el SNF. El estudio de elección se realiza con TC de alta resolución y RM con gadolinio. Ambos exámenes son complementarios para planificar la cirugía y permitir una resección completa y segura del tumor ${ }^{1}$.

Histológicamente son tumores caracterizados por un crecimiento fusiforme con áreas Antoni $A$ y B alternantes, con S-100 positivo en la inmunohistoquímica ${ }^{5-6}$.

El objetivo del tratamiento en los pacientes con SNF debe intentar preservar la función del nervio facial por el mayor tiempo posible. La cirugía y reparación del nervio es el tratamiento de elección en pacientes con alteración de la función nervio facial de grado $\geq I I I$ de House-Brackmann (HB). En casos excepcionales de SNF en pacientes asintomáticos, o sin compromiso del nervio facial (HB grado I 0 II), se prefiere la observación ${ }^{4-7}$.

En dos de nuestros casos clínicos se decidió una resolución quirúrgica, dada la sintomatología de los pacientes y la imposibilidad de excluir otros diagnósticos. En el tercer caso, se decidió mantener una conducta expectante y observación periódica, ya que es un tumor benigno y su resección podría causar parálisis facial invalidante en un paciente joven. Realizar una biopsia intraoperatoria para excluir malignidad es controversial, ya que se asocia a mayor riesgo de parálisis facial ${ }^{3}$.

Los neurinomas de la cuerda del tímpano, a diferencia de los originados en el nervio facial, constituyen una entidad excepcional8-9. El primer caso fue descrito por Nager en 1969 y en la literatura hay sólo 15 casos reportados ${ }^{5-10}$. Se presentan predominantemente en mujeres y en una distri- bución etaria bimodal entre la primera y segunda década y posteriormente entre la quinta y sexta ${ }^{11}$. Se presentan habitualmente con hipoacusia, tinnitus y más raramente parálisis facial. Es interesante que en ninguno de los casos encontrados en la literatura hayan presentado alteración del gusto ${ }^{3}$. La otoscopía es variable pero en general se puede observar un aumento de volumen retrotimpánico en cuadrante posterosuperior ${ }^{12}$. En varios casos reportados, el tumor se extiende a través de la membrana timpánica, ocupando parte del canal auditivo externo ${ }^{13}$.

El tratamiento de elección en este tipo de tumor es resección completa vía transcanal 0 transmastoidea, con desarticulación de la cadena osicular si es necesario $^{13}$. Dado la tendencia de los SNF de ser multicéntricos se debe revisar el trayecto del facial, abarcando como mínimo sus porciones vertical y horizontal ${ }^{14}$.

\section{CONCLUSIÓN}

Los schwannomas del nervio facial y sus ramas son tumores altamente infrecuentes. Habitualmente se presentan con cuadros de sintomatología de larga data y progresión lenta. Actualmente el tratamiento de elección es quirúrgico, con resección completa del tumor, para lo cual el estudio preoperatorio debe incluir una tomografía computarizada e idealmente una resonancia magnética para planificar la cirugía. Sin embargo para la confirmación diagnóstica se debe realizar un estudio anatomopatológico. Estos tumores deben ser considerados dentro del diagnóstico diferencial de los tumores de oído medio. En dos de nuestros pacientes, se observó una ubicación en la porción timpánica del nervio facial y en el otro se observó que el tumor comprometía la cuerda del tímpano. En dos de los pacientes la resección quirúrgica tuvo un resultado favorable y en nuestro tercer caso se decició la observación, debido a que la intervención podría causar parálisis facial invalidante en una persona joven.

\section{BIBLIOGRAFÍA}

1. Chung SY, Kim DI, Lee BH, Yoon PH, Jeon P \& Chung TS. Facial nerve schwannomas: CT and MR findings. Yonsei Med J 1998; 39(2): 148-53.

2. Amano T, Sangatsuda $Y$, Hata N, Inoue D, Mori M, Nakamizo A \& SasakI T. Facial Nerve Schwannoma 
Arising From the Cerebellopontine Angle. Neurologia medico-chirurgica 2013; 53(4): 242-4.

3. Bäck L, Heikkilä T, Passador-Santos F, Saat R, Leivo I \& MÄkITIE AA. Management of facial nerve schwannoma: a single institution experience. Acta Oto-laryngologica 2010; 130(10): 1193-8.

4. Bacciu A, Nusier A, Lauda L, Falcioni M, Russo A \& Sanna $M$. Are the current treatment strategies for facial nerve schwannoma appropriate also for complex cases? Audiology and Neurotology 2013; 18(3): 184-91.

5. Ramírez-Camacho R, Trinidad A, Lois CG, Salas I, de Diego B, Roldán A \& García-Berrocal JR. Clinical and Histological Features of Chorda Tympani Tumors. Journal of International Advanced Otology 2014; 10(1).

6. Browning ST, PhIL M \& Phillips JJ. Pathology in focus. Schwannoma of the chorda tympani nerve. $J$ Laryngol Otol 2000; 114: 81-2.

7. Wilkinson EP, Hoa M, Slattery WH, Fayad JN, Friedman RA, Schwartz MS, \& Brackmann DE. Evolution in the management of facial nerve schwannoma. The Laryngoscope 2011; 121(10): 2065-74.
8. BIGGS ND \& FAGAN PA. Schwannoma of the chorda tympani. The Journal of Laryngology \& Otology 2001; 115(01): 50-2.

9. Undabeitia JI, Undabeitia J, Padilla L \& Municio A. Chorda tympani neuroma. Acta Otorrinolaringologica Española 2013.

10. Nager GT. Acoustm neurinomas' Pathology and differential diagnosis. Arch Otolaryngol 1969; 89: 252-79.

11. Huoh KC \& Cheung SW. Chorda tympani neuroma. Otology \& Neurotology 2010; 31(7): 1172-3.

12. ST BRowIIING, M PHIL, F.R.C.S (ORL-HNS), JJ PHILLIPPS, F.R.C.S, N Wilıiams, F.R. C PАтн. Schwannoma of the chorda tympani nerve. The Journal of Laryngology and Otology 2000; Vol 114: 81-2.

13. Chal $F$, Vanopulos $K$ \& Mcmanus T. Chorda tympani schwannoma. Australian and New Zealand Journal of Surgery 2000; 70(11): 827-8.

14. Magliulo G, D'Amico R, Varacalli $S$ \& CiniglioApPIANI G. Chorda tympani neuroma: diagnosis and management. American Journal of Otolaryngology 2000; 21(1): 65-8. 